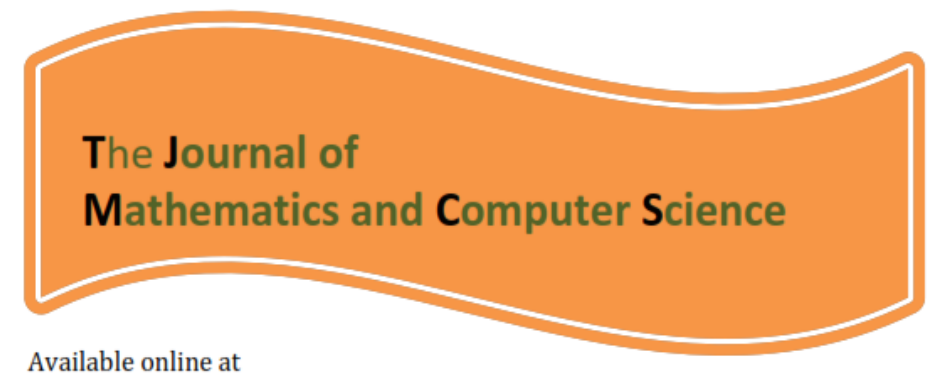

\title{
http://www.TJMCS.com
}

The Journal of Mathematics and Computer Science Vol .3 No.4 (2011) 432 - 439

\section{On critical exponent for the existence and multiplicity of positive weak solutions for a class of $(p, q)$-Laplacian nonlinear system}

\author{
M. B. Ghaemi ${ }^{a}$, G. A. Afrouzi ${ }^{b}$, S. H. Rasouli ${ }^{c}$, M. Choubin ${ }^{d}$ \\ ${ }^{a}$ Department of Mathematics, \\ Iran University of Science and Technology, Narmak, Tehran, Iran \\ e-mail: mghaemi@iust.ac.ir \\ ${ }^{b}$ Department of Mathematics, \\ Faculty of Basic Sciences, Mazandaran University, Babolsar, Iran \\ e-mail: afrouzi@umz.ac.ir \\ ${ }^{c}$ Department of Mathematics, Faculty of Basic Sciences, \\ Babol University of Technology, Babol, Iran \\ e-mail: s.h.rasouli@nit.ac.ir \\ ${ }^{d}$ Department of Mathematics,
}

Faculty of Basic Sciences, Payame Noor University, Tehran, Iran

e-mail: choubin@phd.pnu.ac.ir

Received: August 2011, Revised: November 2011

Online Publication: December 2011

\section{Abstract}

In this paper, we prove the existence of positive weak solution for the nonlinear elliptic system

$$
\begin{cases}-\Delta_{p} u=\lambda_{1} u^{a}+\mu_{1} v^{b}, & x \in \Omega, \\ -\Delta_{q} v=\lambda_{2} u^{c}+\mu_{2} v^{d}, & x \in \Omega, \\ u=0=v, & x \in \partial \Omega,\end{cases}
$$

where $\Delta_{s} z=\operatorname{div}\left(|\nabla z|^{s-2} \nabla z\right), s>1, \lambda_{1}, \lambda_{2}, \mu_{1}$ and $\mu_{2}$ are positive parameters, and $\Omega$ is a bounded domain in $\mathbb{R}^{N}, a+c<p-1$ and $b+d<q-1$. We also discuss a multiplicity result when $0<\lambda_{1}, \lambda_{2}, \mu_{1}, \mu_{2}<\lambda^{*}$ for some $\lambda^{*}$. We obtain our results via the method of sub - and super solutions.

Keywords: Positive weak solution; $p$-Laplacian; Sub - and super solutions.

AMS Subject Classification: 35J55, 35J65. 


\section{Introduction}

Consider the system

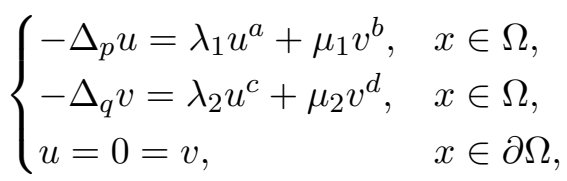

where $\Delta_{s} z=\operatorname{div}\left(|\nabla z|^{s-2} \nabla z\right), s>1, \lambda_{1}, \lambda_{2}, \mu_{1}$ and $\mu_{2}$ are positive parameters, and $\Omega$ is a bounded domain in $\mathbb{R}^{N}$ with smooth boundary $\partial \Omega$. In addition, we assume that $1<p, q<N$.

Chen in [3] discussed the system $-\Delta_{p} u=\lambda u^{a} v^{b},-\Delta_{q} v=\lambda u^{c} v^{d}$ with zero Dirichlet boundary condition. Our goal is somewhat modest: to find an analogue result for problem (1.1). The boundary value problem

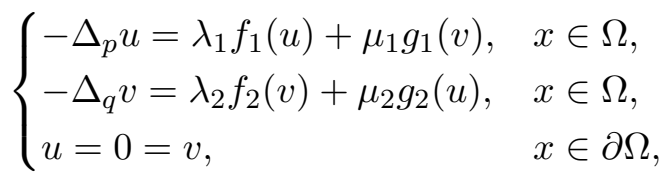

have been studied extensively in recent years (see [1, 6]). Ali and Shivaji [1] studied the existence of positive solution for the problem (1.2) with $\lambda_{1}+\mu_{1}$ and $\lambda_{2}+\mu_{2}$ large when

$$
\lim _{x \rightarrow 0} \frac{g_{1}\left(M\left[g_{2}(x)\right]^{1 / q-1}\right)}{x^{p-1}}=0
$$

for every $M>0, \lim _{x \rightarrow 0} \frac{f_{1}(x)}{x^{p-1}}=0$ and $\lim _{x \rightarrow 0} \frac{f_{2}(x)}{x^{q-1}}=0$. Also we refer to [6] for results on systems related to (1.2) in the case $p=q$ and nonlinearities with falling zeroes.

These problems arise in some physical models and are interesting in applications at combustion, mathematical biology, chemical reactions.

The above assumption for the problem (1.1) implies $b c<(p-1)(q-1)$. In this work, we first prove the existence of positive solution with each positive parameters $\lambda_{1}, \lambda_{2}, \mu_{1}, \mu_{2}$, and next establish the existence of at least two positive solution when $0<\lambda_{1}, \lambda_{2}, \mu_{1}, \mu_{2}<\lambda^{*}$ for some $\lambda^{*}$. Our approach is based on the method of sub- and supersolutions (see $[4,5,7]$ ).

The main results of this paper are Theorems 1.1 and 1.3.

Theorem 1.1. Suppose that $a, d \geq 0, b, c>0, a+c<p-1$ and $b+d<q-1$. Then problem (1.1) has a positive weak solution for each positive parameters $\lambda_{1}, \lambda_{2}, \mu_{1}$ and $\mu_{2}$.

Remark 1.2. By Theorem 1.1 in [1], there exists a positive solution of the problem (1.1) for $\lambda_{1}+\mu_{1}$ and $\lambda_{2}+\mu_{2}$ large. But in our paper, (1.1) has a positive solution for each positive parameters $\lambda_{1}, \lambda_{2}, \mu_{1}$ and $\mu_{2}$.

Theorem 1.3. Suppose that $a, d \geq 0, b, c>0, a+c<p-1$ and $b+d<q-1$. Then there exists $\lambda^{*}>0$ such that for $0<\lambda_{1}, \lambda_{2}, \mu_{1}, \mu_{2}<\lambda^{*}$, (1.1) has at least two positive weak solutions.

\section{$2 \quad$ Proof of Theorem 1.1}

Proof. We shall establish Theorem 1.1 by constructing a positive weak subsolution $\left(\psi_{1}, \psi_{2}\right) \in$ $W_{0}^{1, p} \times W_{0}^{1, q}$ and a supersolution $\left(z_{1}, z_{2}\right) \in W_{0}^{1, p} \times W_{0}^{1, q}$ of (1.1) such that $\psi_{i} \leq z_{i}$ for $i=1,2$. 
That is $, \psi_{i}, z_{i}$ satisfies $\left(\psi_{1}, \psi_{2}\right)=(0,0)=\left(z_{1}, z_{2}\right)$ on $\partial \Omega$,

$$
\begin{gathered}
\int_{\Omega}\left|\nabla \psi_{1}\right|^{p-2} \nabla \psi_{1} \cdot \nabla f_{1} d x \leq \int_{\Omega}\left(\lambda_{1} \psi_{1}^{a}+\mu_{1} \psi_{2}^{b}\right) f_{1} d x, \\
\int_{\Omega}\left|\nabla \psi_{2}\right|^{q-2} \nabla \psi_{2} \cdot \nabla f_{2} d x \leq \int_{\Omega}\left(\lambda_{2} \psi_{1}^{c}+\mu_{2} \psi_{2}^{d}\right) f_{2} d x, \\
\int_{\Omega}\left|\nabla z_{1}\right|^{p-2} \nabla z_{1} \cdot \nabla f_{1} d x \geq \int_{\Omega}\left(\lambda_{1} z_{1}^{a}+\mu_{1} z_{2}^{b}\right) f_{1} d x, \\
\int_{\Omega}\left|\nabla z_{2}\right|^{q-2} \nabla z_{2} \cdot \nabla f_{2} d x \geq \int_{\Omega}\left(\lambda_{2} z_{1}^{c}+\mu_{2} z_{2}^{d}\right) f_{2} d x,
\end{gathered}
$$

for all test functions $f_{1} \in W_{0}^{1, p}$ and $f_{2} \in W_{0}^{1, q}$ with $f_{1}, f_{2} \geq 0$.

Let $\lambda_{p}$ and $\lambda_{q}$ be the first eigenvalue of the problems, respectively,

$$
\begin{array}{ll}
-\Delta_{p} \phi_{p}=\lambda_{p} \phi_{p}^{p-1}, x \in \Omega, & \phi_{p}=0, x \in \partial \Omega, \\
-\Delta_{q} \phi_{q}=\lambda_{q} \phi_{q}^{q-1}, x \in \Omega, & \phi_{q}=0, x \in \partial \Omega,
\end{array}
$$

where $\phi_{p}$ and $\phi_{q}$ denote the corresponding eigenfunctions, respectively, satisfying $\phi_{p}, \phi_{q}>0$ in $\Omega$ and $\left|\nabla \phi_{p}\right|>0,\left|\nabla \phi_{q}\right|>0$ on $\partial \Omega$. Without loss of generality, we let $\left\|\phi_{p}\right\|_{p}=\left\|\phi_{q}\right\|_{q}=1$.

Since $b c<(p-1-a)(q-1-d)$, we can take $k$ such that

$$
\frac{c}{q-1-d}<k<\frac{p-1-a}{b} \text {. }
$$

We shall verify that $\left(\psi_{1}(x), \psi_{2}(x)\right)=\left(\sigma \phi_{p}^{p^{\prime}}, \sigma^{k} \phi_{q}^{q^{\prime}}\right)$ is a subsolution of $(1.1)$, where $p^{\prime}=p /(p-$ $1), q^{\prime}=q /(q-1)$ and $\sigma>0$ is small and specified later. Let the test function $f_{1}(x) \in W_{0}^{1, p}$ with $f_{1}(x) \geq 0$. Then it follows from (2.1) that

$$
\begin{aligned}
\int_{\Omega}\left|\nabla \psi_{1}\right|^{p-2} \nabla \psi_{1} \cdot \nabla f_{1} d x & =\left(a p^{\prime}\right)^{p-1} \int_{\Omega} \phi_{p}\left|\nabla \phi_{p}\right|^{p-2} \nabla \phi_{p} . \nabla f_{1} d x \\
& =\left(a p^{\prime}\right)^{p-1} \int_{\Omega}\left[\left|\nabla \phi_{p}\right|^{p-2} \nabla \phi_{p} \cdot \nabla\left(\phi_{p} f_{1}\right)-\left|\nabla \phi_{p}\right|^{p} f_{1}\right] d x \\
& =\left(a p^{\prime}\right)^{p-1} \int_{\Omega}\left(\lambda_{p} \phi_{p}{ }^{p}-\left|\nabla \phi_{p}\right|^{p}\right) f_{1} d x .
\end{aligned}
$$

Similarly,

$$
\int_{\Omega}\left|\nabla \psi_{2}\right|^{q-2} \nabla \psi_{2} \cdot \nabla f_{2} d x=\left(a^{k} q^{\prime}\right)^{q-1} \int_{\Omega}\left(\lambda_{q} \phi_{q}^{q}-\left|\nabla \phi_{q}\right|^{q}\right) f_{2} d x
$$

for all $f_{2}(x) \in W_{0}^{1, q}$ with $f_{2}(x) \geq 0$. Let $\eta>0, \mu>0$ be such that

$$
\lambda_{p} \phi_{p}{ }^{p}-\left|\nabla \phi_{p}\right|^{p} \leq 0, \quad \lambda_{q} \phi_{q}{ }^{q}-\left|\nabla \phi_{q}\right|^{q} \leq 0, \quad x \in \bar{\Omega}_{\eta},
$$

and $\mu \leq \phi_{p}, \phi_{q} \leq 1$ on $\Omega_{0}=\Omega \backslash \bar{\Omega}_{\eta}$, where $\bar{\Omega}_{\eta}=\{x \in \Omega: d(x, \partial \Omega) \leq \eta\}$. (This is possible since $\phi_{p}=\phi_{q}=0$ and $\left|\nabla \phi_{p}\right|,\left|\nabla \phi_{q}\right|>0$ on $\partial \Omega$.)

We have from (2.3) that

$$
\left(a p^{\prime}\right)^{p-1} \int_{\bar{\Omega}_{\eta}}\left(\lambda_{p} \phi_{p}^{p}-\left|\nabla \phi_{p}\right|^{p}\right) f_{1} d x \leq 0 \leq \int_{\bar{\Omega}_{\eta}}\left(\lambda_{1} \psi_{1}^{a}+\mu_{1} \psi_{2}^{b}\right) f_{1} d x
$$


and

$$
\left(a^{k} q^{\prime}\right)^{q-1} \int_{\bar{\Omega}_{\eta}}\left(\lambda_{q} \phi_{q}^{q}-\left|\nabla \phi_{q}\right|^{q}\right) f_{2} d x \leq 0 \leq \int_{\bar{\Omega}_{\eta}}\left(\lambda_{2} \psi_{1}^{c}+\mu_{2} \psi_{2}^{d}\right) f_{2} d x .
$$

On the other hand, in $\Omega_{0}$ let $r_{1}=(p-1-a) / c, r_{2}=(p-1-a) /(p-1-a-c), s_{1}=$ $(q-1-d) / b, s_{2}=(q-1-d) /(q-1-d-b)$; Note that $\left(1 / r_{1}\right)+\left(1 / r_{2}\right)=1,\left(1 / s_{1}\right)+\left(1 / s_{2}\right)=1$. We have from (2.2) that

$$
\begin{aligned}
& p-1-\frac{a}{r_{1}}-\frac{k b}{r_{2}} \geq p-1-a-k b>0, \\
& k\left(q-1-\frac{d}{s_{2}}\right)-\frac{c}{s_{1}} \geq k(q-1-d)-c>0 .
\end{aligned}
$$

Thus we choose $\sigma>0$ such that

$$
\begin{gathered}
\sigma^{p-1-\frac{a}{r_{1}}-\frac{k b}{r_{2}}} p^{\prime p-1} \lambda_{p} \phi_{p}{ }^{p} \leq \lambda_{1}{ }^{\frac{1}{r_{1}}} \mu_{1}{ }^{\frac{1}{r_{2}}} \mu^{a \delta+q}, \quad x \in \Omega_{0}, \\
\sigma^{k\left(q-1-\frac{d}{s_{2}}\right)-\frac{c}{s_{1}}} q^{q-1} \lambda_{q} \phi_{q}^{q} \leq \lambda_{2}{ }^{\frac{1}{s_{1}}} \mu_{2}{ }^{\frac{1}{s_{2}}} \mu^{d \gamma+p}, \quad x \in \Omega_{0},
\end{gathered}
$$

where $\delta=p /(p-1-a), \gamma=q /(q-1-d)$. Furthermore

$$
\begin{aligned}
& a \delta r_{1}=\frac{a p}{p-1-a-c} \geq \frac{p a}{p-1}=p^{\prime} a, \\
& d \gamma s_{2}=\frac{d q}{q-1-d-b} \geq \frac{q d}{q-1}=q^{\prime} d,
\end{aligned}
$$

and

$$
\begin{aligned}
& p s_{1}=p\left(\frac{q-1-d}{b}\right)>p\left(\frac{c}{p-1-a}\right) \geq \frac{p c}{p-1}=p^{\prime} c, \\
& q r_{2}=q\left(\frac{p-1-a}{c}\right)>q\left(\frac{b}{q-1-d}\right) \geq \frac{q b}{q-1}=q^{\prime} b .
\end{aligned}
$$

These relations and Young inequality show that

$$
\begin{aligned}
& \left(a p^{\prime}\right)^{p-1} \int_{\Omega_{0}}\left(\lambda_{p} \phi_{p}{ }^{p}-\left|\nabla \phi_{p}\right|^{p}\right) f_{1} d x \leq\left(a p^{\prime}\right)^{p-1} \int_{\Omega_{0}} \lambda_{p} \phi_{p}{ }^{p} f_{1} d x \\
& \leq \int_{\Omega_{0}}\left(\lambda_{1}^{\frac{1}{r_{1}}} \sigma^{\frac{a}{r_{1}}} \mu^{a \delta}\right)\left(\mu_{1}^{\frac{1}{r_{2}}} \sigma^{\frac{k b}{r_{2}}} \mu^{q}\right) f_{1} d x \\
& \leq \int_{\Omega_{0}}\left[\frac{\left(\lambda_{1}^{\frac{1}{r_{1}}} \sigma^{\frac{a}{r_{1}}} \mu^{a \delta}\right)^{r_{1}}}{r_{1}}+\frac{\left(\mu_{1}^{\frac{1}{r_{2}}} \sigma^{\frac{k b}{r_{2}}} \mu^{q}\right)^{r_{2}}}{r_{2}}\right] f_{1} d x \\
& \leq \int_{\Omega_{0}}\left[\left(\lambda_{1}^{\frac{1}{r_{1}}} \sigma^{\frac{a}{r_{1}}} \mu^{a \delta}\right)^{r_{1}}+\left(\mu_{1}^{\frac{1}{r_{2}}} \sigma^{\frac{k b}{r_{2}}} \mu^{q}\right)^{r_{2}}\right] f_{1} d x \\
& =\int_{\Omega_{0}}\left(\lambda_{1} \sigma^{a} \mu^{a \delta r_{1}}+\mu_{1} \sigma^{k b} \mu^{q r_{2}}\right) f_{1} d x \\
& \leq \int_{\Omega_{0}}\left(\lambda_{1} \sigma^{a} \phi_{p}^{p^{\prime} a}+\mu_{1} \sigma^{k b} \phi_{q}^{q^{\prime} b}\right) f_{1} d x \\
& =\int_{\Omega_{0}}\left(\lambda_{1} \psi_{1}^{a}+\mu_{1} \psi_{2}^{b}\right) f_{1} d x
\end{aligned}
$$


and

$$
\begin{aligned}
\left(a^{k} q^{\prime}\right)^{q-1} \int_{\Omega_{0}}\left(\lambda_{q} \phi_{q}^{q}-\left|\nabla \phi_{q}\right|^{q}\right) f_{2} d x & \leq\left(a^{k} q^{\prime}\right)^{q-1} \int_{\Omega_{0}} \lambda_{q} \phi_{q}^{q} f_{2} d x \\
& \leq \int_{\Omega_{0}}\left(\lambda_{2}^{\frac{1}{s_{1}}} \sigma^{\frac{c}{s_{1}}} \mu^{p}\right)\left(\mu_{2}{ }^{\frac{1}{s_{2}}} \sigma^{\frac{k d}{s_{2}}} \mu^{d \gamma}\right) f_{2} d x \\
& \leq \int_{\Omega_{0}}\left[\left(\lambda_{2}^{\frac{1}{s_{1}}} \sigma^{\frac{c}{s_{1}}} \mu^{p}\right)^{s_{1}}+\left(\mu_{2}^{\frac{1}{s_{2}}} \sigma^{\frac{k d}{s_{2}}} \mu^{d}\right)^{s_{2}}\right] f_{2} d x \\
& =\int_{\Omega_{0}}\left(\lambda_{2} \sigma^{c} \mu^{p s_{1}}+\mu_{2} \sigma^{k d} \mu^{d s_{2}}\right) f_{2} d x \\
& \leq \int_{\Omega_{0}}\left(\lambda_{2} \sigma^{c} \phi_{p}^{p^{\prime} c}+\mu_{2} \sigma^{k d} \phi_{q}^{q^{\prime} d}\right) f_{2} d x \\
& =\int_{\Omega_{0}}\left(\lambda_{2} \psi_{1}^{c}+\mu_{2} \psi_{2}^{d}\right) f_{2} d x .
\end{aligned}
$$

Thus the $\left(\psi_{1}, \psi_{2}\right)$ is a subsolution of (1.1).

Next, we construct a supersolution of (1.1). Let $e_{p}(x), e_{q}(x)$ be the positive solutions, respectively, of the problems

$$
\begin{array}{ll}
-\Delta_{p} e_{p}=1, x \in \Omega, & e_{p}=0, x \in \partial \Omega, \\
-\Delta_{q} e_{q}=1, x \in \Omega, & e_{q}=0, x \in \partial \Omega .
\end{array}
$$

Let $\left(z_{1}, z_{2}\right):=\left(A e_{p}, B e_{q}\right)$, where the constants $A, B>0$ are large and to be chosen later. Then we have from (2.4) that

$$
\begin{aligned}
& \int_{\Omega}\left|\nabla z_{1}\right|^{p-2} \nabla z_{1} \cdot \nabla f_{1} d x=A^{p-1} \int_{\Omega} f_{1} d x, \\
& \int_{\Omega}\left|\nabla z_{2}\right|^{q-2} \nabla z_{2} \cdot \nabla f_{2} d x=B^{q-1} \int_{\Omega} f_{2} d x .
\end{aligned}
$$

Let $l=\left\|e_{p}\right\|_{\infty}, L=\left\|e_{q}\right\|_{\infty}$. Since $p, q>1, a<p-1, d<q-1$, so that

$$
\lim _{x \rightarrow \infty} \frac{x^{a}}{x^{p-1}}=0=\lim _{x \rightarrow \infty} \frac{x^{d}}{x^{q-1}}, \lim _{x \rightarrow \infty} x^{p-1}=\infty=\lim _{x \rightarrow \infty} x^{q-1} .
$$

These imply that there exist positive large constants A,B such that

$$
A^{p-1} \geq \lambda_{1}(A l)^{a}+\mu_{1}(B L)^{b}, B^{q-1} \geq \lambda_{2}(A l)^{c}+\mu_{2}(B L)^{d} .
$$

Thus

$$
\begin{aligned}
& A^{p-1} \int_{\Omega} f_{1} d x \geq \int_{\Omega}\left(\lambda_{1} z_{1}{ }^{a}+\mu_{1} z_{2}{ }^{b}\right) f_{1} d x, \\
& B^{q-1} \int_{\Omega} f_{2} d x \geq \int_{\Omega}\left(\lambda_{2} z_{1}{ }^{c}+\mu_{2} z_{2}{ }^{d}\right) f_{2} d x,
\end{aligned}
$$

i.e., $\left(z_{1}, z_{2}\right)$ is a supersolution of (1.1) with $z_{i} \leq \psi_{i}$ for $A, B \operatorname{larg}, i=1,2$. Thus, there exists a solution $(u, v)$ of $(1.1)$ with $\psi_{1} \leq u \leq z_{1}, \psi_{2} \leq v \leq z_{2}$. This completes the proof of theorem 1.1. 


\section{Proof of Theorem 1.3}

Proof. To prove Theorem 1.3, we will construct a solution $\left(\psi_{1}, \psi_{2}\right)$, a strict supersolution $\left(\zeta_{1}, \zeta_{2}\right)$, a strict subsolution $\left(\omega_{1}, \omega_{2}\right)$, and a supersolution $\left(z_{1}, z_{2}\right)$ for $(1.1)$ such that $\left(\psi_{1}, \psi_{2}\right) \leq\left(\zeta_{1}, \zeta_{2}\right) \leq$ $\left(z_{1}, z_{2}\right),\left(\psi_{1}, \psi_{2}\right) \leq\left(\omega_{1}, \omega_{2}\right) \leq\left(z_{1}, z_{2}\right)$, and $\left(\omega_{1}, \omega_{2}\right) \not \leq\left(\zeta_{1}, \zeta_{2}\right)$. Then by the three-solution theorem $[2,7],(1.1)$ has a least three distinct solutions $\left(u_{i}, v_{i}\right), i=1,2,3$, such that

$$
\left(u_{1}, v_{1}\right) \in\left[\left(\psi_{1}, \psi_{2}\right),\left(\zeta_{1}, \zeta_{2}\right)\right],\left(u_{2}, v_{2}\right) \in\left[\left(\omega_{1}, \omega_{2}\right),\left(z_{1}, z_{2}\right)\right]
$$

and

$$
\left(u_{3}, v_{3}\right) \in\left[\left(\psi_{1}, \psi_{2}\right),\left(z_{1}, z_{2}\right)\right] \backslash\left(\left[\left(\psi_{1}, \psi_{2}\right),\left(\zeta_{1}, \zeta_{2}\right)\right] \cup\left[\left(\omega_{1}, \omega_{2}\right),\left(z_{1}, z_{2}\right)\right]\right) .
$$

We first note that $\left(\psi_{1}, \psi_{2}\right)=(0,0)$ is a solution (hence a subsolution). As in section 2 , we can always construct a large supersolution $\left(z_{1}, z_{2}\right)$. By the Theorem 1.1 the problem

$$
\begin{cases}-\Delta_{p}{\tilde{\omega_{1}}}=\lambda_{1}{\tilde{\omega_{1}}}^{a}+\mu_{1}{\tilde{\omega_{2}}}^{b}, & x \in \Omega, \\ -\Delta_{q}{\tilde{\omega_{2}}}^{2}=\lambda_{2}{\tilde{\omega_{1}}}^{c}+\mu_{2}{\tilde{\omega_{2}}}^{d}, & x \in \Omega, \\ \tilde{\omega}_{1}=0=\tilde{\omega}_{2}, & x \in \partial \Omega,\end{cases}
$$

has a positive weak solution $\left(\tilde{\omega_{1}}, \tilde{\omega}_{2}\right)$. Let $\vartheta, \alpha>0$ be such that

$$
\frac{c}{q-1}<\vartheta<\frac{p-1}{b},
$$

and

$$
\max \left\{\alpha^{p-1-a}, \alpha^{p-1-\vartheta b}, \alpha^{\vartheta(q-1)-c}, \alpha^{\vartheta(q-1-d)}\right\}<1 .
$$

(This is possible since $b c<(p-1)(q-1))$. Let $\omega_{1}=\alpha \tilde{\omega_{1}}$ and $\omega_{2}=\alpha^{\vartheta} \tilde{\omega_{2}}$. Then for $x \in \Omega$ we have

$$
\begin{aligned}
\int_{\Omega}\left|\nabla \omega_{1}\right|^{p-2} \nabla \omega_{1} \cdot \nabla f_{1} d x & =\alpha^{p-1} \int_{\Omega}\left|\nabla \tilde{\omega}_{1}\right|^{p-2} \nabla \tilde{\omega_{1}} \cdot \nabla f_{1} d x \\
& =\alpha^{p-1} \int_{\Omega}\left(\lambda_{1} \tilde{\omega}_{1}^{a}+\mu_{1} \tilde{\omega}_{2}^{b}\right) f_{1} d x \\
& =\int_{\Omega}\left(\alpha^{p-1-a} \lambda_{1} \omega_{1}^{a}+\alpha^{p-1-\vartheta b} \mu_{1} \omega_{2}^{b}\right) f_{1} d x \\
& <\int_{\Omega}\left(\lambda_{1} \omega_{1}^{a}+\mu_{1} \omega_{2}^{b}\right) f_{1} d x,
\end{aligned}
$$

and

$$
\begin{aligned}
\int_{\Omega}\left|\nabla \omega_{2}\right|^{q-2} \nabla \omega_{2} . \nabla f_{2} d x & =\alpha^{\vartheta(q-1)} \int_{\Omega}\left|\nabla \tilde{\omega_{2}}\right|^{q-2} \nabla \tilde{\omega_{2}} \cdot \nabla f_{2} d x \\
& =\alpha^{\vartheta(q-1)} \int_{\Omega}\left(\lambda_{2}{\tilde{\omega_{1}}}^{c}+\mu_{2}{\tilde{\omega_{2}}}^{d}\right) f_{2} d x \\
& =\int_{\Omega}\left(\alpha^{\vartheta(q-1)-c} \lambda_{2} \omega_{1}^{c}+\alpha^{\vartheta(q-1-d)} \mu_{2} \omega_{2}{ }^{d}\right) f_{2} d x \\
& <\int_{\Omega}\left(\lambda_{2} \omega_{1}^{c}+\mu_{2} \omega_{2}^{d}\right) f_{2} d x .
\end{aligned}
$$


Thus $\left(\omega_{1}, \omega_{2}\right)$ is a strict subsolution of (1.1). Finally we construct the strict supersolution $\left(\zeta_{1}, \zeta_{2}\right)$. Let $e_{p}, e_{q}, l, L$ be as described in section 2 , and

$$
\left(\zeta_{1}, \zeta_{2}\right)=\left(\epsilon \frac{e_{p}}{l}, \epsilon \frac{e_{q}}{L}\right), \quad \lambda^{*}=\min \left\{\frac{\epsilon^{p-1}}{\beta l^{p-1}}, \frac{\epsilon^{p-1}}{\beta^{\prime} l^{p-1}}, \frac{\epsilon^{q-1}}{\beta L^{q-1}}, \frac{\epsilon^{q-1}}{\beta^{\prime} L^{q-1}}\right\}
$$

where $0<\epsilon<1, \beta>1$ and $\beta^{\prime}=\beta /(\beta-1)$. Note that $\left\|\zeta_{1}\right\|_{\infty}=\left\|\zeta_{2}\right\|_{\infty}=\epsilon$. For $0<\lambda_{1}, \lambda_{2}, \mu_{1}, \mu_{2}<$ $\lambda^{*}$ observe that

$$
\begin{aligned}
\frac{\epsilon^{p-1}}{l^{p-1}} & =\frac{\epsilon^{p-1}}{l^{p-1}}\left(\frac{1}{\beta}+\frac{1}{\beta^{\prime}}\right) \\
& =\frac{\epsilon^{p-1}}{\beta l^{p-1}}+\frac{\epsilon^{p-1}}{\beta^{\prime} l^{p-1}} \\
& >\lambda_{1}+\mu_{1} \geq \lambda_{1} \zeta_{1}{ }^{a}+\mu_{1} \zeta_{2}{ }^{b},
\end{aligned}
$$

and

$$
\begin{aligned}
\frac{\epsilon^{q-1}}{L^{q-1}} & =\frac{\epsilon^{q-1}}{L^{q-1}}\left(\frac{1}{\beta}+\frac{1}{\beta^{\prime}}\right) \\
& =\frac{\epsilon^{q-1}}{\beta L^{q-1}}+\frac{\epsilon^{q-1}}{\beta^{\prime} L^{q-1}} \\
& >\lambda_{2}+\mu_{2} \geq \lambda_{2} \zeta_{1}{ }^{c}+\mu_{2} \zeta_{2}{ }^{d} .
\end{aligned}
$$

Using the inequalities (3.3) and (3.4), we have

$$
\begin{aligned}
& \int_{\Omega}\left|\nabla \zeta_{1}\right|^{p-2} \nabla \zeta_{1} \cdot \nabla f_{1} d x=\frac{\epsilon^{p-1}}{l^{p-1}} \int_{\Omega} f_{1} d x>\int_{\Omega}\left(\lambda_{1} \zeta_{1}{ }^{a}+\mu_{1} \zeta_{2}^{b}\right) f_{1} d x, \\
& \int_{\Omega}\left|\nabla \zeta_{2}\right|^{q-2} \nabla \zeta_{2} \cdot \nabla f_{2} d x=\frac{\epsilon^{q-1}}{L^{q-1}} \int_{\Omega} f_{2} d x>\int_{\Omega}\left(\lambda_{2} \zeta_{1}{ }^{c}+\mu_{2} \zeta_{2}{ }^{d}\right) f_{2} d x,
\end{aligned}
$$

for all $f_{1} \in W_{0}^{1, p}, f_{2} \in W_{0}^{1, q}$ with $f_{1}, f_{2}>0$. Thus $\left(\zeta_{1}, \zeta_{2}\right)$ is strict supersolution of (1.1). Here we can choose $\epsilon$ small so that $\left(\omega_{1}, \omega_{2}\right) \not \leq\left(\zeta_{1}, \zeta_{2}\right)$. We note that the proof of Theorem 1.1 we can choose $\left(z_{1}, z_{2}\right)$ large enough that $\left(\zeta_{1}, \zeta_{2}\right) \leq\left(z_{1}, z_{2}\right)$. Thus there exist three solutions $\left(u_{i}, v_{i}\right)$, $i=1,2,3$, such that satisfies (3.1) and (3.2). Since $\left(\psi_{1}, \psi_{2}\right)=(0,0)$ is a solution it may turn out that $\left(u_{1}, v_{1}\right) \equiv\left(\psi_{1}, \psi_{2}\right) \equiv(0,0)$. In any case we have two positive solutions $\left(u_{2}, v_{2}\right)$ and $\left(u_{3}, v_{3}\right)$ and Theorem 1.3 is proven.

\section{References}

[1] Jaffar Ali, R. Shivaji, Positive solutions for a class of p-Laplacian systems with multiple parameters, J. Math. Anall. Appl. 335 (2007), 1013-1019.

[2] Herbert Amann, Fixed point equations and nonlinear eigenvalue problems in ordered Banach spaces, SIAM Rev. 18 (5) (1976) 620-709.

[3] C. Chen, On positive weak solutions for a class of quasilinear elliptic systems, Nonlin. Anal. 62 (2005) 751-756 
M. B. Ghaemi, G. A. Afrouzi, S.H. Rasouli, M. Choubin/ TJMCS Vol .3 No.4 (2011) 432 - 439

[4] P. Drábek, J. Hernandez, Existence and uniqueness of positive solutions for some quasilinear elliptic problem, Nonlin. Anal. TMA, 44 (2001), 189-204.

[5] P. Drábek, P. Krejčí., P. Takáč., Nonlinear Differential Equations, Chapman \& Hall/CRC, 1999.

[6] Eun Kyoung lee, R. Shivaji, Jinglong Ye, Positive solutions for elliptic equations involving nonlinearities with fallig zeroes, Appl. Math. Letters 22 (2009), 846-851.

[7] R. Shivaji, A remark on the existence of three solutions via sub-super solutions, Nonlin. Anal. and Appl., Lecture notes in pure and applied mathematics. 109 (1987), 561-566. 